\title{
Industrial Applications of Data Mining
}

\author{
Gholamreza Nakhaeizadeh \\ Daimler-Benz, Research and Technology 3 \\ Postfach 2360, Ulm, Germany \\ Nakhaeizadeh@dbag . ulm.daimlerbenz.com
}

\begin{abstract}
In the recent years Data Mining has found a lot of applications in industry and commerce. Some aspects of such applications in Marketing, Quality Management and Risk Management will be discussed:
\end{abstract}

\section{Marketing Applications}

Development of a Predictive Modelling process within the project "Customer Relationship Management" (CRM). The aim is to improve the relationship with customers. Among others customer care and marketing activities need to be better adapted to the needs of (prospective) customers. The outcome of the analysis is a predictive model which can be embedded and used in an operative system.

Development of Adequate Information Models supporting Marketing Research to find out the opinion of customers in respect to products and the service quality and to inform the related departments adequately and timely. To this end since some years several data sources have been exploited and data analysis reveals the inability of the existing information models to differentiate significantly between products and service quality respectively. The main task is the development of data mining method providing sufficient information models for products and services.

Development of Forecast Models for Warranty Costs. The main aim is to develop the forecast models for warranty costs that are caused by legal and voluntary regulations. One of the essential targets is the implementation of a highly automated planning process which is capable to improve forecast accuracy. Besides the classical statistical models, other forecasting models based on neural nets are examined.

\section{Quality Management}

Development of an Early Warning System using Data Mining. The aim is to provide methods that allow an early prediction of fault rates. This should enable the initiation of corrective actions as early as possible. This leads to early identification and detection if the quality goals are violated. During the analysis of the domain, several potential applications are identified and the three most important of them are realized. The solutions is embedded in a unified interactive environment. 
3. Risk management

Increasing globalization efforts in international trade and high fluctuations makes risk analysis of financial markets an inevitable feature of corporate risk management. Given the large interest of the treasury department of companies for the future development of interest rates and exchange rates, the main emphasis is laid on the development of robust and reliable quantitative forecasting methods for currencies, interest rates and yield curves, respectively 